\title{
Change in mental health after smoking cessation: systematic review and meta-analysis
}

The authors of this Research paper, Taylor and colleagues, have alerted us to an error in their study (BMJ 2014;348:g1151, doi:10.1136/bmj.g1151). The meta-analysis included three papers by Kahler and colleagues, and the authors mistakenly mixed up two of these studies when classifying them as general or psychiatric populations. This misclassification affected the sensitivity analysis comparing clinical subgroups and some of the associated text, as outlined below, but did not affect the conclusions or findings of the study.

Firstly, some of the entries in table 8 are incorrect. The values in the "Effect estimate standardised mean difference" column under the "Depression" heading should have been $-0.23(-0.37$ to -0.09 ) for "General" and -0.53 ( -0.77 to -0.29 ) for
"Psychiatric condition." These incorrect figures were also reported in the main text of the results section- they appear in the second paragraph under the headings "Additional analyses, Comparison of clinical population."

Lastly, in appendix 2 of the data supplement, the entry in the "Population" column should have read Psychiatric (not General) for the first of the studies listed by Kahler (Kahler 2002), and General (not Psychiatric) for the second listed study (Kahler 2011).

Cite this as: BMJ 2014;348:g2216

๑ BMJ Publishing Group Ltd 2014 